\title{
SAMPL7 TrimerTrip host-guest binding affinities from extensive alchemical and end-point free energy calculations
}

\author{
Zhe Huai ${ }^{1}$, Huaiyu Yang ${ }^{2}$, Xiao Li $^{3}$, and Zhaoxi Sun ${ }^{1,4 *}$ \\ ${ }^{1}$ State Key Laboratory of Precision Spectroscopy, School of Physics and Electronic Science, East China Normal \\ University, Shanghai 200062, China \\ ${ }^{2}$ College of Engineering, Hebei Normal University, Shijiazhuang 050024, China \\ ${ }^{3}$ Physics, engineering, earth, environment, mechanics (PhITEM), University Grenoble Alpes, 38000 Grenoble, France \\ ${ }^{4}$ Beijing National Laboratory for Molecular Sciences, Institute of Theoretical and Computational Chemistry, College \\ of Chemistry and Molecular Engineering, Peking University, Beijing 100871, China
}

*To whom correspondence should be addressed: z.sun@pku.edu.cn

\begin{abstract}
The prediction of host-guest binding affinities with computational modelling is still a challenging task. In the $7^{\text {th }}$ statistical assessment of the modeling of proteins and ligands (SAMPL) challenge, a new host named TrimerTrip was synthesized and the thermodynamic parameters of 16 structurally diverse guests binding to the host were characterized. In the TrimerTrip-guest challenge, only structures of the host and the guests are provided, which indicates that the predictions of both the binding poses and the binding affinities are under assessment. In this work, starting from the binding poses obtained from our previous enhanced sampling simulations in the configurational space, we perform extensive alchemical and end-point free energy calculations to calculate the host-guest binding affinities retrospectively. The alchemical predictions with two widely accepted charge schemes (i.e. AM1-BCC and RESP) are in good agreement with the experimental reference, while the end-point estimates perform poorly in reproducing the experimental binding affinities. Aside from the absolute value of the binding affinity, the rank of binding free energies is also crucial in drug design. Surprisingly, the end-point MM/PBSA method seems very powerful in reproducing the experimental rank of binding affinities. Although the length of our simulations is long and the intermediate spacing is dense, the convergence behavior is not very good, which may arise from the flexibility of the host molecule. Enhanced sampling techniques in the configurational space may be required to obtain fully converged sampling. Further, as the length of sampling in alchemical free energy calculations already achieves several hundred ns, performing direct simulations of the binding/unbinding event in the
\end{abstract}


physical space could be more useful and insightful. More details about the binding pathway and mechanism could be obtained in this way. The nonequilibrium method could also be a nice choice if one insists to use the alchemical method, as the intermediate sampling is avoided to some extent. 


\section{Introduction}

Understanding the interaction network in host-guest systems is at the center of drug discovery. Proteinligand binding is a special case of host-guest interactions. The model host-guest systems are smaller and simpler than the protein-ligand complexes. Specifically, the interaction network and thus the binding/unbinding pathway in the host-guest complexes are simpler. Further, the host-guest binding affinities are comparable to the protein-ligand ones. Therefore, they serve as nice candidates to validate the experimental and computational protocols. ${ }^{1-4}$ The statistical assessment of the modeling of proteins and ligands (SAMPL) challenges provide a series of challenging systems to assess the accuracy and efficiency of the computational modeling in various cases, e.g. solvation free energies, pKa, and protein-ligand binding, host-guest binding, and partition coefficients..$^{3,5-9}$ The SAMPL challenges often include macrocyclic and rigid hosts and drug-like guests. For the host-guest binding affinity predictions in the past SAMPL challenges, computational modelling generally provides predictions with about $2 \mathrm{kcal} / \mathrm{mol}$ mean deviations from the experimental references. ${ }^{3,8}$

The thermodynamic determinant of the host-guest binding is the free energy difference between different states. ${ }^{10-14}$ Various methods have been developed to calculate the free energy difference between the bound and unbound states. The alchemical method enables feasible calculations of the free energy difference between different states. ${ }^{15-20}$ The procedure is quite simple to apply and the computational cost is affordable, as only one collective variable (CV) or order parameter is used to describe the alchemical transformation. The correct calculation of the free energy difference requires the accurate estimation of the partition functions or their ratio. The class of estimators relying on this relation includes free energy perturbation $(\mathrm{FEP})^{21}$ and its variants, including the Gaussian approximated $\mathrm{FEP}^{22,23}$ and acceptance ratio methods of BAR, ${ }^{24,}{ }^{25} \mathrm{UBAR},{ }^{26} \mathrm{RBAR},{ }^{26}$ and MBAR.${ }^{27,}{ }^{28}$ Among these methods, BAR and MBAR are statistically optimal and thus are of the highest efficiency. ${ }^{12,13,25,29-34}$ For a given dataset of energetic values, they converge better than the other methods. Alternatively, under reasonable approximations, other easier calculation methods are proposed. Integration methods integrate the ensemble averages of the derivatives of the Hamiltonians numerically. Although they are theoretically rigorous when the integration step is infinitely small, in practical application approximations (i.e. systematic biases) are introduced due to the finite size of the integration step. The methods are often called thermodynamic integration (TI $)^{35-37}$ and the variants of TI are named according to their integration methods. The trapezoid rule is the most popular method for numerical integration. If the transformation is performed reversibly, FEP and TI reduce to the slow growth TI, where the reversible work in the transformation is used to estimate the free energy difference. ${ }^{38-40}$ The 
nonequilibrium scenarios of FEP and BAR are Jarzynski's Identity (JI) ${ }^{31,41}$ and Crooks' Equation (CE). ${ }^{42}$ The difference between them is that the instantaneous work is used in FEP and BAR while in JI and CE the nonequilibrium work accumulated in nonequilibrium pulling is used. Approximated methods include semiempirical extensions of linear interaction energy $y^{43-46}$ and quasi-equilibrium linear response approximation. ${ }^{46,}$ ${ }^{47}$ Further extending the approximation leads to coarser methods such as the end-point free energy simulation methods of $\mathrm{MM} / \mathrm{PBSA}^{48}$ and MM/GBSA. ${ }^{49}$ These methods are very efficient in computing the free energy difference but are of less accuracy. ${ }^{50-54}$ Their most efficient and widely used scheme is the single-trajectory regime, which avoids the need for converging the fluctuation of the intra-molecular energies. Practically, in free energy calculations in drug discovery, these less detailed methods are often employed to screen the probably useful candidates, after which comprehensive free energy calculations with the alchemical method are applied to refine the dataset and find the most useful ones. The alchemical and end-point free energy calculations are efficient when the binding affinity is the only thermodynamic quantity of interest. These methods avoid the simulation of intermediate states in the binding/unbinding process and thus enhance the convergence. However, this is where the weakness lies. The details about the binding/unbinding pathway are unknown. Further, the simulation with the unbiased technique only explores the neighborhood of the starting configurations, which could lead to systematic bias that may not be eliminated in finite-time simulations. ${ }^{17,55-58}$ Biasing the sampling in the configurational space could partially eliminate this initial-configuration-related bias, but the selection of efficient CVs is also a challenging task. ${ }^{56,59-61}$

In the newest SAMPL 7 challenge, the newly added TrimerTrip host is included and 16 structurally diverse guest molecules are synthesized. The detailed thermodynamic parameters are measured with isothermal titration calorimetry experiments. ${ }^{1}$ This challenge includes only the structures of the host and the guest, ${ }^{62}$ which indicates that the computational study should initiate from the prediction of possible binding poses. In our previous work, starting from randomly selected configurations, we employ the threedimensional (3D) spherical-coordinate- $(\rho, \theta, \varphi) \mathrm{CV}$ set to perform direct sampling of the binding/unbinding event in the host-guest systems. ${ }^{63}$ The spherical coordinates enable a scan of the relative position of the two components of the complex (i.e. the host and the guest). ${ }^{64,65}$ In principle, the binding poses of the host-guest systems obtained from previous spherical-coordinates-biased simulations are more reliable than docking, as all-atom free-energy-weighted simulations are involved. Therefore, in the current work, these binding poses are used as the starting point of the end-point and alchemical free energy calculations. Two widely accepted charge schemes for small molecules are employed to construct the atomic 
charges of the host and the guests. Note that the current work begins after the close of the SAMPL7 challenge. Thus, the results are not formally submitted to the challenge and the calculations are retrospective.

\section{Methodology and Computational Details}

System preparation. A central glycoluril trimer and two triptycene caps form the host TrimerTrip. The 16 guests targeting the host in the SAMPL7 challenge are simulated. In Fig. 1, we present an illustration of the host-guest binding and the chemical structures of the 16 guest molecules. The protonation states of the guest molecules are adjusted according to the experimental reference. ${ }^{1}$ The experimental observation shows that there is no significant self-association for this host. ${ }^{1}$ Therefore, in our simulation, the simulated systems are constructed according to the one-to-one binding protocol. Two widely accepted charge schemes including AM1-BCC ${ }^{66}$ and RESP are used to parameterize the host and the guests. We use the singleconformation scheme in charge fitting and the configuration used in the charge generating procedure is the guest part of the first structure of each host-guest complex obtained from our previous work at https://github.com/proszxppp/SAMPL7_TTP. Namely, in the GitHub repository for each host-guest system there are 6 binding poses, and we extract the first pose, remove the host part and use the coordinates of the guest to perform the AM1-BCC and RESP charge fitting procedure. The general Amber force field $(\mathrm{GAFF})^{67}$ force field is used to obtain the other missing force field parameters. The starting configuration in the alchemical free energy calculation is obtained from the previous enhanced sampling simulations ${ }^{63}$ at https://github.com/proszxppp/SAMPL7 TTP. In our previous work, we employed 3D spherical coordinates as the $\mathrm{CV}$ set to bias the simulation and used metadynamics to enhance the sampling efficiency. The structures extracted from the global free energy minimum are the binding poses deposited in the online repository. The guest $\mathrm{g} 8$ is used as the reference guest in the relative free energy calculation, and the starting configuration of the host-g8 complex is shown in Fig. 1. This structure of the host molecule is used in the parameterization of the host. As this structure differs from the one used for parameterization of the host in the previous spherical-coordinates-biased simulations, ${ }^{63}$ the binding thermodynamics would differ from the previous work. The end-point free energy calculation starts from the first binding pose of each complex provided at the online repository https://github.com/proszxppp/SAMPL7 TTP. Each complex is solvated with TIP $3 \mathrm{P}^{68,69}$ water molecules with the minimum distance between the box edge and the surface of the complex to be $12 \AA$. Periodicity is treated with periodic boundary conditions. Non-polarizable spherical counter ions $\mathrm{s}^{70,71}$ of $\mathrm{Na}^{+}$are added for neutralization. 
Alchemical free energy calculation. We perform the widely used relative free energy calculations to estimate the relative binding affinities of different guest molecules. The reference guest is set to g8. The hostg8 structure shown in Fig. 1 is used as the starting configuration for simulations of the solvated complex. The guest $\mathrm{g} 8$ in this structure is gradually mutated to another guest in the alchemical transformation. We superpose the other guest on g8 and manually alter the coordinates to maximize the similarity between the host-gust complex and the binding poses obtained from our previous work in the online repository. The difference between binding affinities could be estimated with the difference between the free energy change for mutating g8 to another guest in the solvated complex and that in solvent. The staging technique is used to enhance the convergence of the simulation. 21 equally spaced alchemical windows are used in each transformation. Namely, $\Delta \lambda=0.05, \lambda_{\min }=0.00$, and $\lambda_{\max }=1.00$. The charge and $\mathrm{vdW}$ transformations are performed in a single-step fashion with the nonlinear separation-shifted softcore potential ${ }^{39,72-75}$ with the default parameters in AMBER and the linear mixing rule. The whole guest is included in the softcore region. In some cases, the softcore potential could lead to problematic energetics in the alchemical transformation. However, in our case, we do not observe any strange behavior of the host-guest systems. The simulation in each alchemical intermediate starts from 4000 cycles minimization with $5 \mathrm{kcal} / \mathrm{mol} / \mathrm{A}^{2}$ restraints on non-hydrogen atoms of solute, 6000 cycles minimization without any restraint, $1 \mathrm{~ns}$ NVT heating from $0 \mathrm{~K}$ to $298 \mathrm{~K}$ and $2 \mathrm{~ns}$ NPT equilibration. Later, $20 \mathrm{~ns}$ sampling with a sampling interval of $2 \mathrm{ps}$ is performed. The resulting overall simulation time for each alchemical mutation is about $483 \mathrm{~ns}$, and the production run is $420 \mathrm{~ns}$. Thus, the overall computational cost for each relative free energy calculation includes $483 \mathrm{~ns}$ sampling for solvated complex and $483 \mathrm{~ns}$ sampling for solvated ligands. To extract the results from the alchemical simulations, we used the statistically efficient perturbation estimator $\mathrm{MBAR}^{27,28}$ and the integration method TI. The autocorrelation time of the partial derivative of alchemical Hamiltonian $\left.\frac{\partial H}{\partial \lambda}\right|_{\lambda=\lambda_{i}}$ is calculated to extract independent samples for rigorous uncertainty estimation. ${ }^{12,13,30,76,77}$ The statistical error or the standard deviation (SD) is obtained from the analytical formula of the free energy estimator.

When the Ewald method is used to treat the electrostatic interactions, a change of the net charge during alchemical mutations introduces artifacts. The finite-size effects introduce a non-negligible error in the electrostatic potential energy calculations. This correction term could be calculated from various methods. ${ }^{78-}$ ${ }^{81}$ In the current work, we employ the charge correction approach developed by Rocklin et al. ${ }^{80}$ to estimate the electrostatic correction term in the alchemical transformation between guests with different net charges. The correction term includes the following 4 terms: the periodicity-induced net-charge interactions, the 
periodicity-induced net-charge undersolvation, the discrete solvent effects, and the residual integrated potential effects. We employ the APBS program $^{82}$ in the PB calculations of the last term.

End-point free energy calculation. The end-point methods of MM/PBSA and MM/GBSA ${ }^{50}$ are also used to calculate the binding affinities. The starting structures are the first binding poses provided in the online repository in our previous work. For each host-guest complex, we performed 4000 cycles minimization with $5 \mathrm{kcal} / \mathrm{mol} / \mathrm{A}^{2}$ restraints on non-hydrogen atoms of solute, 6000 cycles minimization without any restraint, 1 ns NVT heating and 2 ns NPT equilibration, after which 100 ns production run with a sampling interval of $100 \mathrm{ps}$ is performed. This sampling interval is much longer than the normally used ones. Thus, we take the obtained data points as uncorrelated samples. These independent configurations are then post-processed to calculate the gas-phase enthalpic changes and the changes of solvation free energies upon the host-guest binding. We use the MM/PBSA and MM/GBSA protocols in the end-point calculations. The GB model used in the GBSA calculation is the $\mathrm{GB}^{\mathrm{OBC}}$ model ${ }^{83,84} \mathrm{~GB}$ is a computationally efficient approximated form of $\mathrm{PB}$ and thus the GB calculation is less computationally demanding. The computational costs of these end-point estimators are much smaller than the configurational sampling and these end-point estimates are computationally cheaper than the alchemical method. The entropic contribution in the host-guest binding could be estimated with various methods, e.g. the normal mode analysis, ${ }^{85}$ the quasi-harmonic method, ${ }^{86,87}$ or the recently developed interaction entropy method. ${ }^{88-90}$ However, as the errors introduced in the treatment of solvation are often much larger than the entropic contributions and including the entropic component only leads to limited accuracy improvement, we do not perform the entropy calculations in the current work.

In all MD simulations, the $\mathrm{SHAKE}^{91}$ algorithm is used to constrain bonds involving hydrogen atoms, ${ }^{92}$ and the time step for integrating the equations of motion is set to $2 \mathrm{fs}$. Langevin dynamics ${ }^{93}$ with the collision frequency of $5 \mathrm{ps}^{-1}$ are implemented for temperature regulation. The cutoff for non-bonded interactions in the real space is set to $10 \AA$, and the PME method is used to treat the long-range electrostatics. ${ }^{94,95}$ The GPU version of the pmemd engine with the hybrid precision (SPFP) in the AMBER $18^{96}$ suite is used to perform MD simulations. Note that the current work starts after the close of the SAMPL7 challenge. Therefore, we do not participate in the SAMPL7 challenge and the current results are not available on the online repository of the SAMPL7 challenge.

\section{Result and discussion}

The first thing to check in free energy calculations is the convergence. As the spacing of the alchemical windows in our simulations is already dense, we then just check the time-dependence of the free energy 
estimates. In Fig. S2, the time-evolutions of the free energy estimates under two charge schemes are presented. If the fluctuation of the free energy estimate is small in the last part of the sampling, the convergence behavior of the free energy simulation is good. The threshold of a small fluctuation could be $0.5 \mathrm{kcal} / \mathrm{mol}$, which seems impossible to be achieved for many systems in the current work. Therefore, for some systems (e.g. the g8->g15 transformation) the convergence behavior is good, while there are some cases (e.g. g8->g5) that the free energy estimates seem still un-converged at the end of the production run. The reference alchemical free energy calculations provided on the SAMPL7 online server also reports the convergence problem on this host. ${ }^{97}$ Note that our simulation with 21 windows and $23 \mathrm{~ns}$ sampling in each window is long in modern alchemical free energy calculations. In previous SAMPL challenges such as the SAMPL6 challenge, the relative free energy calculations used about 11 alchemical windows and several ns (e.g. $3 \mathrm{~ns}$ ) sampling in each intermediate. ${ }^{2}$ The resulting overall simulation time for each alchemical transformation is about an order of magnitude shorter than the current work. Therefore, our results could be used to approximate the long-time estimates of alchemical free energy calculation with the unbiased sampling technique. The systems with convergence problems may need enhanced sampling methods to aid the sampling in the configurational space and obtain fully converged estimates. We further visualize the trajectories to check the fluctuation of the complexes. In our previous work with enhanced sampling techniques in the configurational space, the fluctuation of the TrimerTrip pocket is observed to be large. ${ }^{63}$ The TrimerTrip host in the current unbiased simulation experiences some degrees of fluctuations, but the magnitude is relatively small, which also indicates the poor convergence behavior of the sampling. We align the structures of the host-guest complexes at the end of alchemical and end-point simulations with the initial configuration, and these structures overlap significantly, which indicates that the structural fluctuations during equilibrium simulations are limited. Further, when the sampling achieves such a time scale (i.e. $~ 500$ ns), performing alchemical free energy calculation is already not a computationally efficient choice. Note that the calculation of each relative binding affinity includes two parts of simulations (i.e. solvated complex and solvated ligands), which further increases the computational cost. Direct simulations of the binding/unbinding event with enhanced sampling techniques could converge within such a time scale, and much more details about the binding/unbinding pathway and mechanism could be obtained. The nonequilibrium technique could be useful in dealing with this convergence problem of the alchemical method, as the sampling in the intermediate states is avoided to some extent. As the guests are structurally diverse, the perturbation introduced in the alchemical free energy calculation could be large in some cases. As a result, performing relative alchemical free energy calculation could be not an efficient choice. When 
the two end points of the alchemical pathway have little structural similarity, absolute free energy calculation could be a more suitable choice to improve the convergence. The end-point free energy calculations also employ a long-sampling-time protocol (i.e. $100 \mathrm{~ns}$ sampling and 100 ps sampling interval). Therefore, the end-point results could also be used as the long-time estimates.

In Table 1 and 2, the predicted binding affinities from alchemical free energy calculations are presented. We use the mean signed error (MSE), the mean absolute error (MAE), the root-mean-squared error (RMSE) to assess the deviations of the predicted binding affinities from the experimental reference. To compare the consistency of the predicted ranks of binding affinities and the experimental one, we calculate the Kendall $\tau$ rank coefficient ${ }^{98}$ and the Pearlman's predictive index (PI). ${ }^{99}$ For these ranking coefficients, 1.0 means perfect correct prediction, -1.0 is perfect incorrect prediction, and 0.0 is random. Under both charge schemes, the error estimates are about $2.5-3 \mathrm{kcal} / \mathrm{mol}$. Therefore, RMSE and other mean deviations are comparable to the SAMPL6 cases. ${ }^{3,8,64}$ The ranking coefficients are about 0.2 , which indicates that the predicted ranks show some similarity with the experimental reference, but the consistency is not good. Interestingly, although the error estimates of TI are comparable to the MBAR ones, the ranking coefficients of TI are better than MBAR. Upon convergence, these two methods should provide identical results, which also indicates that the degree of convergence in the current alchemical free energy calculations is not very good. The integration error (i.e. the systematic bias introduced in numerical integration) could be another source of error triggering the discrepancy between TI and MBAR estimates. The results obtained with RESP charges are better than the AM1-BCC ones, but the improvement is very small. In Fig. 2a-b, the comparisons between the free energy estimates obtained with AM1-BCC and RESP charges are presented. With the alchemical method (TI and MBAR), for some systems, the AM1-BCC results agree better with the experiment, while in the other the RESP predictions are better. Neither method is consistently better than the other in all cases. Overall, the error estimates and the ranking coefficients are only improved a little with RESP charges.

These error estimates are larger than the ones reported in our previous work. One possible reason is that the free energy estimates obtained with the alchemical method have convergence problems. As a result, the current estimates may have a systematic bias (e.g. initial-configuration-induced bias) and thus are not directly comparable to our previous predictions with enhanced sampling techniques in the configurational space. ${ }^{63}$ Another possible reason is the difference between the configurations used in parameterizing the host and the guests. The structure of the host used in the current parameterization is the one in the host-g8 bound structure obtained from our previous work. This difference leads to different atomic charges and thus 
different outcomes of the simulation.

As for the end-point free energy calculations, the detailed results are summarized in Table S1 and S2, and the relative binding affinities are given in Table 1 and 2. As no end-point estimate is reported for the TrimerTrip-guest systems in the SAMPL7 challenge, ${ }^{97}$ our calculation fills this cap by providing the only available end-point free energy results, enabling a rational evaluation of the end-point methods. For both charge schemes, the MM/PBSA method outperforms the MM/GBSA one. With AM1-BCC charges, RMSE of MM/PBSA is about $1.4 \mathrm{kcal} / \mathrm{mol}$ smaller than the MM/GBSA one. The ranking coefficients of MM/PBSA are all larger than zero, which indicates that the predicted rank is in agreement with the experimental one to some extent. By contrast, the ranking coefficients obtained from MM/GBSA are near-zero, which suggests that the predicted rank shows random behavior and is uncorrelated with the experimental reference. When the RESP method is used to parameterize the systems, significant improvements are observed, especially for the MM/PBSA method. As shown in the comparison between the MM/PBSA predictions with two charge schemes in Fig. 2c, the RESP estimates are smaller than the AM1-BCC ones and are closer to the experimental values in most cases. As for MM/GBSA in Fig. 2d, the RESP results are also smaller than the AM1-BCC ones, but both methods still predict binding affinities far from the experimental reference. The RMSE of the MM/PBSA estimates decreases by about $3 \mathrm{kcal} / \mathrm{mol}$, and the ranking coefficients are also improved significantly. The ranking prediction with MM/PBSA and RESP charges is the best one among all protocols employed in the current work. The MM/GBSA estimates improve about $1 \mathrm{kcal} / \mathrm{mol}$ for RMSE and about 0.3 for the ranking metric PI with RESP charges. Note that our observation about the relative performance of these two charge models in end-point free energy calculations is not unique. From the data reported in the Gibb Deep Cavity Cavitands (GDCC) dataset of the SAMPL7 host-guest challenges (Table 3 of the reference), ${ }^{97}$ the RESP-better-than-BCC phenomenon could also be observed. In that case, for the end-point MM/PBSA estimates, the RMSE of RESP results is smaller than the BCC one, which indicates that RESP is better than BCC.

The RESP-smaller-than-BCC phenomenon mentioned above should be discussed in more detail. This is not a general behavior but a system-specific property, and the origin of this phenomenon should be investigated. As shown in Table S1 and S2, with either of the end-point methods, the absolute binding affinities under different charge schemes are similar for some systems, while in other cases some differences are observed. The reference g8 calculation is one of the latter cases. We do not observe the RESP-smallerthan-BCC behavior for the absolute binding affinities. Therefore, this behavior originates from the calculation of the binding affinity of the reference guest $\mathrm{g} 8$ or those of the other guests, as these two parts 
are included in the calculation of the relative binding affinity. As the reference binding affinities obtained from the two charge models show obvious differences and the other cases show relatively small chargemodel-dependence, the phenomenon should arise from the reference calculation. As the two charge models perform similarly in the theoretically rigorous alchemical free energy calculation, the thermodynamics described with the two charge schemes should be similar. In some cases, there are statistically significant differences between RESP and BCC estimates with the alchemical method. However, the RESP-smallerthan-BCC trend is generally absent. As the AM1-BCC model is fitted to reproduce the electrostatic potential (ESP) obtained from ab initio calculations and the ESP data is used in the RESP fitting, the deviation of the ESP produced by AM1-BCC charges from the RESP one should be the reason for the difference of binding affinities with different charge models. When the theoretically not-so-rigorous end-point methods are used, approximations and systematic errors are introduced. Specifically, the systematic error introduced by the approximated end-point calculation method in the reference calculation differs from the other guests, and the situations are different under the two charge models. As a result, the relative binding affinities calculated from this reference value have this relative systematic error. There could be two origins of this systematic error. The first one is the approximated end-point calculation procedure, which ignores the changes of the conformational ensemble upon binding/unbinding. The second one is the implicit solvent used to treat the changes of the solvation free energy. The charges used in the current work are compatible with explicit solvents, while the implicit solvent models may not provide sufficient electrostatic screening.

The prediction-experiment correlations are presented in Fig. 3. The alchemical estimates (TI and MBAR) scatter around the $y=x$ line, while the end-point estimates (MM/PBSA and MM/GBSA) show significant deviations. The MM/PBSA estimates with AM1-BCC charges seem to consistently overestimate the binding affinities, and the MM/GBSA ones also have this tendency. However, the end-point estimates with RESP charges do not have this behavior, which indicates that the RESP charges could perform better when dealing with host-guest systems.

Finally, some comments on the comparison between the calculated results in the current work and the submissions in the TrimerTrip challenge should be added. Several submissions based on fixed-charge force fields in the TrimerTrip challenge employed the alchemical method. Reference calculations with the equilibrium Hamiltonian replica exchange framework starting from binding poses obtained from docking (Docking/GAFF/YANK_REF and Docking/GAFF/YANK_REF_2 in the reference) ${ }^{97}$ were performed, providing predictions with $\sim 7 \mathrm{kcal} / \mathrm{mol}$ RMSE. This error is much larger than the current calculations, which should arise from the selection of initial conformations. As our initial configurations are obtained 
from atomistic simulations augmented by enhanced sampling techniques, they are in principle more reliable than the poses obtained from docking. Another alchemical calculation employing the nonequilibrium framework also started from the docking procedure, but later it used the replica exchange method with solute tempering to equilibrate the docked poses and obtain Boltzmann-weighted canonical configurations. The predictions from this nonequilibrium alchemical free energy simulations achieve an RMSE similar to the current case, ${ }^{97}$ which indicates that fixed-charge models could provide estimates of similar accuracies to the current work. Therefore, the starting configuration of alchemical free energy calculations plays a key role in the correct calculation of the binding thermodynamics. Robust pose-searching and refinement methods are also required.

\section{Conclusion}

In this work, we performed alchemical and end-point free energy calculations on the TrimerTrip-guest complexes. The binding poses obtained from our previous enhanced sampling simulations in the configurational space are used as the starting structures. Two widely accepted charge schemes are used to describe the host and the guests. The predicted binding affinities with the alchemical method are in agreement with the experimental reference, while the end-point methods provide predictions with significant deviations. For the alchemical method, RMSE and MAE are about $2.5 \mathrm{kcal} / \mathrm{mol}$ and MSE is close to zero. By contrast, the end-point ones are much larger. These observations are similar to the SAMPL6 cases. Surprisingly, the end-point MM/PBSA method outperforms the other methods in the prediction of the rank of binding affinities. The performance of the RESP charge scheme is better than the AM1-BCC one. The improvement is small for the alchemical method, but is remarkable when using the MM/PBSA method. Another interesting observation is that the convergence behavior of the TrimerTrip-guest systems is not very good. Although our alchemical free energy simulation employs dense intermediate spacing $(\Delta \lambda=0.05)$ and long simulation times in each intermediate ( $23 \mathrm{~ns})$, the free energy estimates seem still un-converged. This phenomenon may arise from the flexibility of the host molecule and enhanced sampling techniques in the configurational space may be required to get fully converged results. Further, the computational cost of the current alchemical free energy calculation is high (i.e. sampling time $\sim 500 \mathrm{~ns}$ ). Note that the relative free energy calculation includes two parts (i.e. solvated complex and solvated ligands), which further increases the computational cost. With these computational resources, directly simulating the binding/unbinding event in the physical space could be more insightful, as more details about the binding pathway and mechanism could be obtained. Another choice could be the nonequilibrium method. Coupling 
it with the alchemical method could help the sampling problem to some extent, as the extensive sampling in the intermediate states is avoided.

\section{Acknowledgement}

This work is supported by China Postdoctoral Science Foundation. Dr. Zhaoxi Sun is supported by the PKU-Boya Postdoctoral Fellowship. We are grateful for many valuable and insightful comments on the performance of different charge schemes from the anonymous reviewers.

\section{Conflicts of interest}

There are no conflicts of interest to declare.

\section{Supporting information}

The time evolutions of the binding affinities obtained with the alchemical free energy calculation and the detailed free energy components of MM/PBSA and MM/GBSA estimates are given in the supporting information. 


\section{References}

1. Ndendjio, S. Z.; Liu, W.; Yvanez, N.; Meng, Z.; Zavalij, P. Y.; Isaacs, L., Triptycene walled glycoluril trimer: synthesis and recognition properties. New J. Chem. 2020, 44, 338-345.

2. Caldararu, O.; Olsson, M. A.; Ignjatović, M. M.; Wang, M.; Ryde, U., Binding free energies in the SAMPL6 octa-acid host-guest challenge calculated with MM and QM methods. Journal of computer-aided molecular design 2018, 32 , 1027-1046.

3. Rizzi, A.; Murkli, S.; McNeill, J. N.; Yao, W.; Sullivan, M.; Gilson, M. K.; Chiu, M. W.; Isaacs, L.; Gibb, B. C.; Mobley, D. L., Overview of the SAMPL6 host-guest binding affinity prediction challenge. Journal of Computer-Aided Molecular Design 2018, 32, 937-963.

4. Eken, Y.; Patel, P.; Díaz, T.; Jones, M. R.; Wilson, A. K., SAMPL6 host-guest challenge: binding free energies via a multistep approach. Journal of computer-aided molecular design 2018, 32, 1097-1115.

5. Mobley, D. L.; Wymer, K. L.; Lim, N. M.; Guthrie, J. P., Blind prediction of solvation free energies from the SAMPL4 challenge. Journal of computer-aided molecular design 2014, 28, 135-50.

6. Monroe, J. I.; Shirts, M. R., Converging free energies of binding in cucurbit[7]uril and octa-acid host-guest systems from SAMPL4 using expanded ensemble simulations. Journal of computer-aided molecular design 2014, 28, 401-15.

7. Song, L. F.; Bansal, N.; Zheng, Z;; Merz, K. M., Detailed potential of mean force studies on host-guest systems from the SAMPL6 challenge. Journal of computer-aided molecular design 2018, 32, 1013-1026.

8. Procacci, P.; Guarrasi, M.; Guarnieri, G., SAMPL6 host-guest blind predictions using a non equilibrium alchemical approach. Journal of computer-aided molecular design 2018, 32, 965-982.

9. Nishikawa, N.; Han, K.; Wu, X.; Tofoleanu, F.; Brooks, B. R., Comparison of the umbrella sampling and the double decoupling method in binding free energy predictions for SAMPL6 octa-acid host-guest challenges. Journal of Computer-Aided Molecular Design 2018, 32, 1075-1086.

10. Sun, Z.; Zhang, J. Z. H., Thermodynamic Insights of Base Flipping in TNA Duplex: Force Fields, Salt Concentrations, and Free-Energy Simulation Methods. CCS Chemistry 2020, 2, 1026-1039.

11. Wang, X.; Xingzhao, T.; Boming, D.; John Z. H., Z.; Sun, Z., BAR-based Optimum Adaptive Steered MD for Configurational Sampling. J. Comput. Chem. 2019, 40, 1270-1289.

12. Wang, X.; Tu, X.; Zhang, J. Z. H.; Sun, Z., BAR-based Optimum Adaptive Sampling Regime for Variance Minimization in Alchemical Transformation: The Nonequilibrium Stratification. Phys. Chem. Chem. Phys. 2018, 20, 2009-2021.

13. Sun, Z. X.; Wang, X. H.; Zhang, J. Z. H., BAR-based Optimum Adaptive Sampling Regime for Variance Minimization in Alchemical Transformation. Phys. Chem. Chem. Phys. 2017, 19, 15005-15020.

14. Sun, Z.; Wang, X.; Zhang, J. Z. H.; He, Q., Sulfur-substitution-induced base flipping in the DNA duplex. Phys. Chem. Chem. Phys. 2019, 21, 14923-14940.

15. Swope, W. C., A computer simulation method for the calculation of equilibrium constants for the formation of physical clusters of molecules: Application to small water clusters. J. Chem. Phys. 1982, 76, 637.

16. Pham, T. T.; Shirts, M. R., Identifying low variance pathways for free energy calculations of molecular transformations in solution phase. J. Chem. Phys. 2011, 135, 034114.

17. Procacci, P.; Chelli, R., Statistical Mechanics of Ligand-Receptor Noncovalent Association, Revisited: Binding Site and Standard State Volumes in Modern Alchemical Theories. J. Chem. Theory Comput. 2017, 13, 1924-1933.

18. Sun, Z:; Wang, X.; Zhang, J. Z., Theoretical understanding of the thermodynamics and interactions in transcriptional regulator TtgR-ligand binding. Phys. Chem. Chem. Phys. 2020, 22, 1511-1524.

19. Sun, Z;; Wang, X.; Zhao, Q.; Zhu, T., Understanding Aldose Reductase-Inhibitors interactions with free energy simulation. Journal of Molecular Graphics and Modelling 2019, 91, 10-21.

20. Wang, X.; Sun, Z., Understanding PIM-1 kinase inhibitor interactions with free energy simulation. Phys. Chem. Chem. Phys. 2019, 21, 7544-7558.

21. Zwanzig, R. W., High Temperature Equation of State by A Perturbation Method. J. Chem. Phys. 1954, 22, 1420-1426.

22. Hummer, G., Fast-growth thermodynamic integration: Error and efficiency analysis. J. Chem. Phys. 2001, 114, 73307337. 
23. Eastwood, M. P.; Hardin, C.; Luthey-Schulten, Z.; Wolynes, P. G., Statistical mechanical refinement of protein structure prediction schemes: Cumulant expansion approach. J. Chem. Phys. 2002, 117, 4602-4615.

24. Shirts, M. R.; Bair, E.; Hooker, G.; Pande, V. S., Equilibrium Free Energies from Nonequilibrium Measurements using Maximum-likelihood Methods. Physical review letters 2003, 91, 140601.

25. Bennett, C. H., Efficient Estimation of Free Energy Differences from Monte Carlo data. J. Comput. Phys. 1976, 22, 245268.

26. Fenwick, M. K.; Escobedo, F. A., On the use of Bennett's acceptance ratio method in multi-canonical-type simulations. J. Chem. Phys. 2004, 120, 3066-74.

27. Tan, Z., On a Likelihood Approach for Monte Carlo Integration. J. Am. Stat. Assoc. 2004, 99, $1027-1036$.

28. Shirts, M. R.; Chodera, J. D., Statistically optimal analysis of samples from multiple equilibrium states. J. Chem. Phys. 2008, 129, 124105.

29. Wang, X.; Sun, Z., A Theoretical Interpretation of Variance-based Convergence Citeria in Perturbation-based Theories. arXiv preprint arXiv:1803.03123 2018.

30. Sun, Z.; Wang, X.; Song, J., Extensive Assessment of Various Computational Methods for Aspartate's pKa Shift. J. Chem. Inf. Model. 2017, 57, 1621-1639.

31. Jarzynski, C., Equilibrium free-energy differences from nonequilibrium measurements: A master-equation approach. Phys. Rev. E 1997, 56, 5018-5035.

32. Wang, X.; Sun, Z., Determination of Base Flipping Free Energy Landscapes from Nonequilibrium Stratification. J. Chem. Inf. Model. 2019, 59, 2980-2994.

33. Wang, X.; He, Q.; Sun, Z., BAR-Based Multi-Dimensional Nonequilibrium Pulling for Indirect Construction of a QM/MM Free Energy Landscape. Phys. Chem. Chem. Phys. 2019, 21, 6672-6688

34. Sun, Z., BAR-based multi-dimensional nonequilibrium pulling for indirect construction of QM/MM free energy landscapes: from semi-empirical to ab initio. Phys. Chem. Chem. Phys. 2019, 21, 21942-21959

35. Bruckner, S.; Boresch, S., Efficiency of alchemical free energy simulations. II. Improvements for thermodynamic integration. J. Comput. Chem. 2011, 32, 1320-1333.

36. Resat, H.; Mezei, M., Studies on free energy calculations. I. Thermodynamic integration using a polynomial path. J. Chem. Phys. 1993, 99, 6052-6061.

37. Resat, H.; Mezei, M., Studies on free energy calculations. II. A theoretical approach to molecular solvation. J. Chem. Phys. 1994, 101, 6126-6140.

38. Kirkwood, J. G., Statistical Mechanics of Fluid Mixtures. J. Chem. Phys. 1935, 3, 300.

39. Pearlman, D. A.; Kollman, P. A., The lag between the Hamiltonian and the system configuration in free energy perturbation calculations. Journal of Chemical Physics 1989, 91, 7831-7839.

40. Straatsma, T. P.; Mccammon, J. A., Treatment of rotational isomers in free energy calculations. II. Molecular dynamics simulation study of 18-crown-6 in aqueous solution as an example of systems with large numbers of rotational isomeric states. J. Chem. Phys. 1989, 91, 3631-3637.

41. Jarzynski, C., A Nonequilibrium Equality for Free Energy Differences. Physical Review Letters 1997, 78, $2690-2693$.

42. Crooks, G. E., Nonequilibrium Measurements of Free Energy Differences for Microscopically Reversible Markovian Systems. J. Stat. Phys. 1998, 90, 1481-1487.

43. Aqvist, J., .; Medina, C., .; Samuelsson, J. E., A new method for predicting binding affinity in computer-aided drug design. Protein Eng. 1994, 7, 385-91.

44. Carlson, H. A.; Jorgensen, W. L., An Extended Linear Response Method for Determining Free Energies of Hydration. Journal of Physical Chemistry 1995, 99, 10667-10673.

45. Wang, W.; Wang, J.; Kollman, P. A., What determines the van der Waals coefficient $\beta$ in the LIE (linear interaction energy) method to estimate binding free energies using molecular dynamics simulations? Proteins-structure Function \& Bioinformatics 1999, 34, 395-402.

46. Leach, A. R., Molecular Modeling Principles \& Applications. Pearson education 2001

47. Lee, F. S.; Chu, Z. T.; Bolger, M. B.; Warshel, A., Calculations of antibody-antigen interactions: microscopic and semi$15 / 27$ 
microscopic evaluation of the free energies of binding of phosphorylcholine analogs to McPC603. Protein Eng. 1992, 5, 215-28.

48. Ferrari, A. M.; Degliesposti, G.; Sgobba, M.; Rastelli, G., Validation of an automated procedure for the prediction of relative free energies of binding on a set of aldose reductase inhibitors. Biorg. Med. Chem. 2007, 15, 7865-7877.

49. Rapp, C.; Kalyanaraman, C.; Schiffmiller, A.; Schoenbrun, E. L.; Jacobson, M. P., A Molecular Mechanics Approach to Modeling Protein-Ligand Interactions: Relative Binding Affinities in Congeneric Series. J. Chem. Inf. Model. 2011, 51, 2082-9.

50. Miller, B. R.; Mcgee, T. D.; Swails, J. M.; Homeyer, N.; Gohlke, H.; Roitberg, A. E., MMPBSA.py: an efficient program for end-state free energy calculations. J Chem Theory Comput 8:3314-3321. J. Chem. Theory Comput. 2012, 8, 3314-3321.

51. Honig, B.; Nicholls, A., Classical electrostatics in biology and chemistry. Science 1995, 268, 1144-9.

52. Su, P. C.; Tsai, C. C.; Mehboob, S.; Hevener, K. E.; Johnson, M. E., Comparison of radii sets, entropy, QM methods, and sampling on MM-PBSA, MM-GBSA, and QM/MM-GBSA ligand binding energies of $F$. tularensis enoyl-ACP reductase ( $F$ abl). J. Comput. Chem. 2015, 36, 1859-1873.

53. Sangpheak, W.; Khuntawee, W.; Wolschann, P.; Pongsawasdi, P.; Rungrotmongkol, T., Enhanced stability of a naringenin/2, 6-dimethyl $\beta$-cyclodextrin inclusion complex: Molecular dynamics and free energy calculations based on MM-and QM-PBSA/GBSA. Journal of Molecular Graphics and Modelling 2014, 50, 10-15.

54. Tsitsanou, K. E.; Hayes, J. M.; Keramioti, M.; Mamais, M.; Oikonomakos, N. G.; Kato, A.; Leonidas, D. D.; Zographos, S. E., Sourcing the affinity of flavonoids for the glycogen phosphorylase inhibitor site via crystallogra phy, kinetics and QM/MM-PBSA binding studies: comparison of chrysin and flavopiridol. Food and chemical toxicology 2013, 61, 14-27. 55. Moraca, F.; Amato, J.; Ortuso, F.; Artese, A.; Pagano, B.; Novellino, E.; Alcaro, S.; Parrinello, M.; Limongelli, V., Ligand binding to telomeric G-quadruplex DNA investigated by funnel-metadynamics simulations. Proc. Natl. Acad. Sci. U.S.A. 2017, 114, E2136-E2145.

56. Valsson, O.; Tiwary, P.; Parrinello, M., Enhancing Important Fluctuations: Rare Events and Metadynamics from a Conceptual Viewpoint. Annual Review of Physical Chemistry 2016, 67, 159.

57. Tiwary, P.; Limongelli, V.; Salvalaglio, M.; Parrinello, M., Kinetics of protein-ligand unbinding: Predicting pathways, rates, and rate-limiting steps. Proc. Natl. Acad. Sci. U.S.A. 2015, 112, 386-91.

58. Huang, N.; Kalyanaraman, C.; Bernacki, K.; Jacobson, M. P., Molecular mechanics methods for predicting proteinligand binding. Phys. Chem. Chem. Phys. 2006, 8, 5166-77.

59. Mendels, D.; Piccini, G.; Parrinello, M., Collective variables from local fluctuations. J. Phys. Chem. Lett. 2018, 9, 27762781.

60. Tiwary, P.; van de Walle, A., Accelerated molecular dynamics through stochastic iterations and collective variable based basin identification. Phys. Rev. B 2013, 87, 094304.

61. Hashemian, B.; Arroyo, M., Modeling and enhanced sampling of molecular systems with smooth and nonlinear data driven collective variables. J. Chem. Phys. 2013, 139, 214101.

62. https://github.com/samplchallenges/SAMPL7/tree/master/host_guest/Isaacs_clip.

63. Sun, Z., SAMPL 7 TrimerTrip Host-Guest Binding Poses and Binding Affinities from Spherical-Coordinates-Biased Simulations. 2020.

64. Sun, Z.; He, Q.; Li, X.; Zhu, Z., SAMPL6 host-guest binding affinities and binding poses from spherical-coordinatesbiased simulations. Journal of Computer-Aided Molecular Design 2020, 34, 589-600.

65. Capelli, R.; Carloni, P.; Parrinello, M., Exhaustive Search of Ligand Binding Pathways via Volume-based Metadynamics. J. Phys. Chem. Lett. 2019, 10, 3495-3499.

66. Jakalian, A.; Jack, D. B.; Bayly, C. I., Fast, efficient generation of high-quality atomic charges. AM1-BCC model: II. Parameterization and validation. J. Comput. Chem. 2002, 23, 1623-41.

67. Wang, J.; Wolf, R. M.; Caldwell, J. W.; Kollman, P. A.; Case, D. A., Development and testing of a general amber force field. J. Comput. Chem. 2004, 25, 1157-1173.

68. Jorgensen, W. L.; Chandrasekhar, J.; Madura, J. D.; Impey, R. W.; Klein, M. L., Comparison of Simple Potential Functions for Simulating Liquid Water. J. Chem. Phys. 1983, 79, 926-935. 
69. Price, D. J.; Brooks III, C. L., A Modified TIP3P Water Potential for Simulation with Ewald Summation. J. Chem. Phys. 2004, 121, 10096-10103.

70. Joung, I. S.; Cheatham III, T. E., Determination of Alkali and Halide Monovalent Ion Parameters for Use in Explicitly Solvated Biomolecular Simulations. J. Phys. Chem. B 2008, 112, 9020-9041.

71. Joung, I. S.; Cheatham, T. E., Molecular Dynamics Simulations of the Dynamic and Energetic Properties of Alkali and Halide Ions Using Water-Model-Specific Ion Parameters. J. Phys. Chem. B 2009, 113, 13279-13290.

72. Steinbrecher, T.; Mobley, D. L.; Case, D. A., Nonlinear scaling schemes for Lennard-Jones interactions in free energy calculations. J. Chem. Phys. 2007, 127, 214108.

73. Zacharias, M.; Straatsma, T. P.; Mccammon, J. A., Separation-shifted scaling, a new scaling method for Lennard-Jones interactions in thermodynamic integration. J. Chem. Phys. 1994, 100, 9025-9031.

74. Beutler, T. C.; Mark, A. E.; Schaik, R. C. V.; Gerber, P. R.; Gunsteren, W. F. V., Avoiding singularities and numerical instabilities in free energy calculations based on molecular simulations. Chemical Physics Letters 1994, 222, $529-539$.

75. Levitt, M., Protein folding by restrained energy minimization and molecular dynamics. Journal of Molecular Biology 1983, 170, 723-64.

76. Klimovich, P. V.; Shirts, M. R.; Mobley, D. L., Guidelines for the Analysis of Free Energy Calculations. Journal of Computer-Aided Molecular Design 2015, 29, 397-411.

77. Chodera, J. D.; Swope, W. C.; Pitera, J. W.; Seok, C.; Dill, K. A., Use of the Weighted Histogram Analysis Method for the Analysis of Simulated and Parallel Tempering Simulations. J. Chem. Theory Comput. 2007, 3, $26--41$.

78. Chen, W.; Deng, Y.; Russell, E.; Wu, Y.; Abel, R.; Wang, L., Accurate Calculation of Relative Binding Free Energies between Ligands with Different Net Charges. J. Chem. Theory Comput. 2018, 14, 6346-6358.

79. Morgan, B. R.; Massi, F., Accurate Estimates of Free Energy Changes in Charge Mutations. J. Chem. Theory Comput. 2010, 6, 1884-1893.

80. Rocklin, G. J.; Mobley, D. L.; Dill, K. A.; Hünenberger, P. H., Calculating the binding free energies of charged species based on explicit-solvent simulations employing lattice-sum methods: An accurate correction scheme for electrostatic finite-size effects. J. Chem. Phys. 2013, 139, 184103.

81. Lin, Y.-L.; Aleksandrov, A.; Simonson, T.; Roux, B., An Overview of Electrostatic Free Energy Computations for Solutions and Proteins. J. Chem. Theory Comput. 2014, 10, 2690-2709.

82. Baker, N. A.; Sept, D.; Joseph, S.; Holst, M. J.; McCammon, J. A., Electrostatics of nanosystems: Application to microtubules and the ribosome. Proc. Natl. Acad. Sci. USA 2001, 98, 10037-10041.

83. Onufriev, A.; Bashford, D.; Case, D. A., Exploring protein native states and large-scale conformational changes with a modified generalized born model. Proteins Structure Function \& Bioinformatics 2004, 55, 383-94.

84. Feig, M.; Onufriev, A.; Lee, M. S.; Im, W.; Case, D. A., Performance comparison of generalized born and Poisson methods in the calculation of electrostatic solvation energies for protein structures. J. Comput. Chem. 2004, 25, 265-84.

85. Case, D. A., Normal mode analysis of protein dynamics. Curr. Opin. Struct. Biol. 2010, 4, $285-290$.

86. Chang, C. E.; Chen, W.; Gilson, M. K., Evaluating the Accuracy of the Quasiharmonic Approximation. J. Chem. Theory Comput. 2005, 1.

87. Karplus, M.; Kushick, J. N., Method for estimating the configurational entropy of macromolecules. Macromolecules 1981, 14, 325-332.

88. Qiu, L.; Yan, Y.; Sun, Z.; Song, J.; Zhang, J. Z., Interaction entropy for computational alanine scanning in proteinprotein binding. Wiley Interdisip. Rev. Comput. Mol. Sci. 2018, 8, e1342.

89. Sun, Z.; Yan, Y. N.; Yang, M.; Zhang, J. Z., Interaction Entropy for Protein-Protein Binding. J. Chem. Phys. 2017, 146, 124124.

90. Duan, L.; Liu, X.; Zhang, J. Z., Interaction entropy: a new paradigm for highly efficient and reliable computation of protein-ligand binding free energy. Journal of the American Chemical Society 2016, 138, 5722-5728.

91. Ryckaert, J. P.; Ciccotti, G.; Berendsen, H. J. C., Numerical Integration of The Cartesian Equations of Motion of A System with Constraints: Molecular Dynamics of n -alkanes. J. Comput. Phys. 1977, 23, 327-341.

92. Miyamoto, S.; Kollman, P. A., Settle: An Analytical Version of The SHAKE and RATTLE Algorithm for Rigid Water $17 / 27$ 
Models. J. Comput. Chem. 1992, 13, 952-962.

93. Pastor, R. W.; Brooks, B. R.; Szabo, A., An analysis of the accuracy of Langevin and molecular dynamics algorithms. Molecular Physics 1988, 65, 1409-1419.

94. York, D. M.; Darden, T. A.; Pedersen, L. G., The Effect of Long-range Electrostatic Interactions in Simulations of Macromolecular Crystals: A Comparison of The Ewald and Truncated List Methods. J. Chem. Phys. 1993, 99, 8345-8348. 95. Tuckerman, M. E.; Berne, B. J.; Martyna, G. J., Molecular dynamics algorithm for multiple time scales: Systems with long range forces. J. Chem. Phys. 1991, 94, 6811-6815.

96. Case, D. A.; Cheatham, T. E.; Tom, D.; Holger, G.; Luo, R.; Merz, K. M.; Alexey, O.; Carlos, S.; Bing, W.; Woods, R. J., The Amber Biomolecular Simulation Programs. J. Comput. Chem. 2005, 26, 1668-1688.

97. Martin, A.; David, M., SAMPL 7 Challenge Overview: Assessing the Reliability of Polarizable and Non-Polarizable Methods for Host-Guest Binding Free Energy Calculations. 2020.

98. Kendall, M. G., A New Measure of Rank Correlation. Biometrika 1938, 30, 81-93.

99. Pearlman, D. A.; Charifson, P. S., Are Free Energy Calculations Useful in Practice? A Comparison with Rapid Scoring Functions for the p38 MAP Kinase Protein System. Journal of Medicinal Chemistry 2001, 44, 3417-3423. 
Table 1. The TrimerTrip-guest binding free energies (in kcal/mol) computed via various methods ( $\Delta \Delta G_{T I}$, $\Delta \Delta G_{M B A R}, \Delta \Delta G_{\mathrm{MM} / \mathrm{PBSA}}$, and $\left.\Delta \Delta G_{\mathrm{MM} / \mathrm{GBSA}}\right)$ with the AM1-BCC charges and the corresponding experimental values $\left(\Delta G_{E X P}, \Delta \Delta G_{E X P}\right)$. The results of $\mathrm{g} 6, \mathrm{~g} 9, \mathrm{~g} 11$ and $\mathrm{g} 12$ are presented after the electrostatic correction. All values are in $\mathrm{kcal} / \mathrm{mol}$. MSE, MAE, RMSE, $\tau$ and PI serve as quality measurements. The guest $\mathrm{g} 8$ is used as reference and thus its values are all zeros.

\begin{tabular}{ccccccccccc}
\hline Guest & $\Delta G_{\text {EXP }}$ & $\Delta \Delta G_{\text {EXP }}$ & $\Delta \Delta G_{\mathrm{TI}}$ & $\mathrm{SD}$ & $\Delta \Delta G_{\mathrm{MBAR}}$ & $\mathrm{SD}$ & $\Delta \Delta G_{\mathrm{MM} / \mathrm{PBSA}}$ & $\mathrm{SD}$ & $\Delta \Delta G_{\mathrm{MM} / \mathrm{GBSA}}$ & $\mathrm{SD}$ \\
\hline $\mathrm{g} 1$ & -6.1 & 3.4 & 2.6 & 0.3 & 0.9 & 0.6 & 7.6 & 0.3 & 1.7 & 0.3 \\
$\mathrm{~g} 2$ & -8.3 & 1.1 & -1.5 & 0.3 & -3.1 & 0.8 & 4.9 & 0.3 & 1.8 & 0.3 \\
g3 & -10.1 & -0.6 & -2.2 & 0.2 & -0.9 & 0.5 & 0.3 & 0.2 & -0.5 & 0.3 \\
g5 & -11.1 & -1.7 & -1.2 & 0.3 & -0.9 & 0.5 & 3.0 & 0.3 & 1.6 & 0.2 \\
g6 & -9.6 & -0.2 & -3.2 & 0.6 & -2.8 & 0.4 & 7.0 & 0.2 & 14.6 & 0.3 \\
g7 & -6.5 & 3.0 & 2.0 & 0.4 & 2.0 & 0.8 & 6.4 & 0.3 & -1.6 & 0.3 \\
g8 & -9.5 & 0.0 & 0.0 & 0.0 & 0.0 & 0.0 & 0.0 & 0.0 & 0.0 & 0.0 \\
g9 & -7.6 & 1.9 & 3.5 & 0.5 & 3.2 & 0.4 & 11.5 & 0.2 & 14.7 & 0.3 \\
g10 & -8.2 & 1.3 & -2.2 & 0.4 & -2.5 & 0.7 & 4.2 & 0.3 & -0.9 & 0.3 \\
g11 & -9.0 & 0.4 & 0.0 & 0.5 & -0.1 & 0.4 & 6.7 & 0.3 & 10.4 & 0.3 \\
g12 & -8.3 & 1.2 & -1.2 & 0.5 & -1.2 & 0.3 & 9.2 & 0.2 & 11.0 & 0.2 \\
g15 & -10.5 & -1.1 & -4.8 & 0.3 & -5.2 & 0.4 & 10.3 & 0.2 & 15.8 & 0.2 \\
g16 & -11.5 & -2.1 & 2.5 & 0.3 & 2.3 & 0.4 & 4.2 & 0.3 & 5.0 & 0.3 \\
g17 & -11.8 & -2.4 & 3.6 & 0.4 & 4.2 & 0.5 & 6.1 & 0.3 & 7.3 & 0.3 \\
g18 & -10.6 & -1.1 & -0.5 & 0.3 & -3.0 & 0.5 & 15.3 & 0.2 & 8.4 & 0.2 \\
g19 & -11.7 & -2.3 & -7.7 & 0.3 & -7.2 & 0.5 & -1.9 & 0.2 & 4.8 & 0.2 \\
\hline RMSE & & & 3.0 & & 3.2 & & 7.2 & & 8.6 & -5.8 \\
MSE & & & 0.7 & & 0.9 & & -5.9 & & 6.9 &
\end{tabular}


Table 2. The TrimerTrip-guest binding free energies (in $\mathrm{kcal} / \mathrm{mol}$ ) computed via various methods ( $\Delta \Delta G_{T I}$, $\Delta \Delta G_{M B A R}, \Delta \Delta G_{\mathrm{MM} / \mathrm{PBSA}}$, and $\left.\Delta \Delta G_{\mathrm{MM} / \mathrm{GBSA}}\right)$ with the RESP charges and the corresponding experimental values $\left(\Delta G_{E X P}, \Delta \Delta G_{E X P}\right)$. The results of $\mathrm{g} 6, \mathrm{~g} 9, \mathrm{~g} 11$ and $\mathrm{g} 12$ are presented after the electrostatic correction. All values are in $\mathrm{kcal} / \mathrm{mol}$. MSE, MAE, RMSE, $\tau$ and PI serve as quality measurements. The guest $\mathrm{g} 8$ is used as reference and thus its values are all zeros.

\begin{tabular}{|c|c|c|c|c|c|c|c|c|c|c|}
\hline Guest & $\Delta G_{\mathrm{EXP}}$ & $\Delta \Delta G_{\mathrm{EXP}}$ & $\Delta \Delta G_{\mathrm{TI}}$ & $\mathrm{SD}$ & $\Delta \Delta G_{\mathrm{MBAR}}$ & $\mathrm{SD}$ & $\Delta \Delta G_{\mathrm{MM} / \mathrm{PBSA}}$ & $\mathrm{SD}$ & $\Delta \Delta G_{\mathrm{MM} / \mathrm{GBSA}}$ & $\mathrm{SD}$ \\
\hline g1 & -6.1 & 3.4 & 5.9 & 0.2 & 4.4 & 0.7 & 11.4 & 0.2 & 15.7 & 0.2 \\
\hline g2 & -8.3 & 1.1 & 0.3 & 0.2 & 1.1 & 0.9 & -0.1 & 0.2 & -2.4 & 0.2 \\
\hline g3 & -10.1 & -0.6 & -2.4 & 0.2 & -1.9 & 0.9 & -4.5 & 0.2 & -5.6 & 0.2 \\
\hline g5 & -11.1 & -1.7 & -1.4 & 0.2 & -0.8 & 0.6 & -0.7 & 0.2 & -2.5 & 0.2 \\
\hline g6 & -9.6 & -0.2 & -2.3 & 0.6 & -2.0 & 0.4 & -0.5 & 0.2 & -7.8 & 0.2 \\
\hline g7 & -6.5 & 3.0 & 6.7 & 0.2 & 7.4 & 0.5 & 6.7 & 0.2 & -3.5 & 0.2 \\
\hline g8 & -9.5 & 0.0 & 0.0 & 0.0 & 0.0 & 0.0 & 0.0 & 0.0 & 0.0 & 0.0 \\
\hline g9 & -7.6 & 1.9 & 0.7 & 0.5 & -0.5 & 0.4 & 6.0 & 0.2 & -0.5 & 0.2 \\
\hline g10 & -8.2 & 1.3 & -0.3 & 0.2 & -2.1 & 0.6 & 1.6 & 0.3 & -13.6 & 0.3 \\
\hline g11 & -9.0 & 0.4 & -0.3 & 0.5 & -1.5 & 0.6 & 1.3 & 0.2 & -2.2 & 0.2 \\
\hline g12 & -8.3 & 1.2 & -2.7 & 0.4 & -2.9 & 0.3 & 1.7 & 0.2 & -3.3 & 0.2 \\
\hline g15 & -10.5 & -1.1 & -1.4 & 0.3 & -1.8 & 0.4 & 0.3 & 0.2 & -6.2 & 0.2 \\
\hline g16 & -11.5 & -2.1 & 1.5 & 0.4 & 1.8 & 0.7 & 1.7 & 0.2 & 1.6 & 0.2 \\
\hline g17 & -11.8 & -2.4 & 2.9 & 0.3 & 2.9 & 0.5 & 1.8 & 0.2 & 2.3 & 0.2 \\
\hline g18 & -10.6 & -1.1 & 3.9 & 0.4 & 1.5 & 0.5 & 6.7 & 0.2 & -1.4 & 0.2 \\
\hline g19 & -11.7 & -2.3 & -5.0 & 0.5 & -5.6 & 0.5 & -11.3 & 0.2 & -21.0 & 0.2 \\
\hline RMSE & & & 2.8 & & 2.8 & & 4.3 & & 7.7 & \\
\hline MSE & & & -0.3 & & 0.0 & & -1.3 & & 3.2 & \\
\hline MAE & & & 2.2 & & 2.3 & & 3.1 & & 5.8 & \\
\hline$\tau$ & & & 0.2 & & 0.0 & & 0.4 & & -0.1 & \\
\hline PI & & & 0.4 & & 0.3 & & 0.7 & & 0.3 & \\
\hline
\end{tabular}



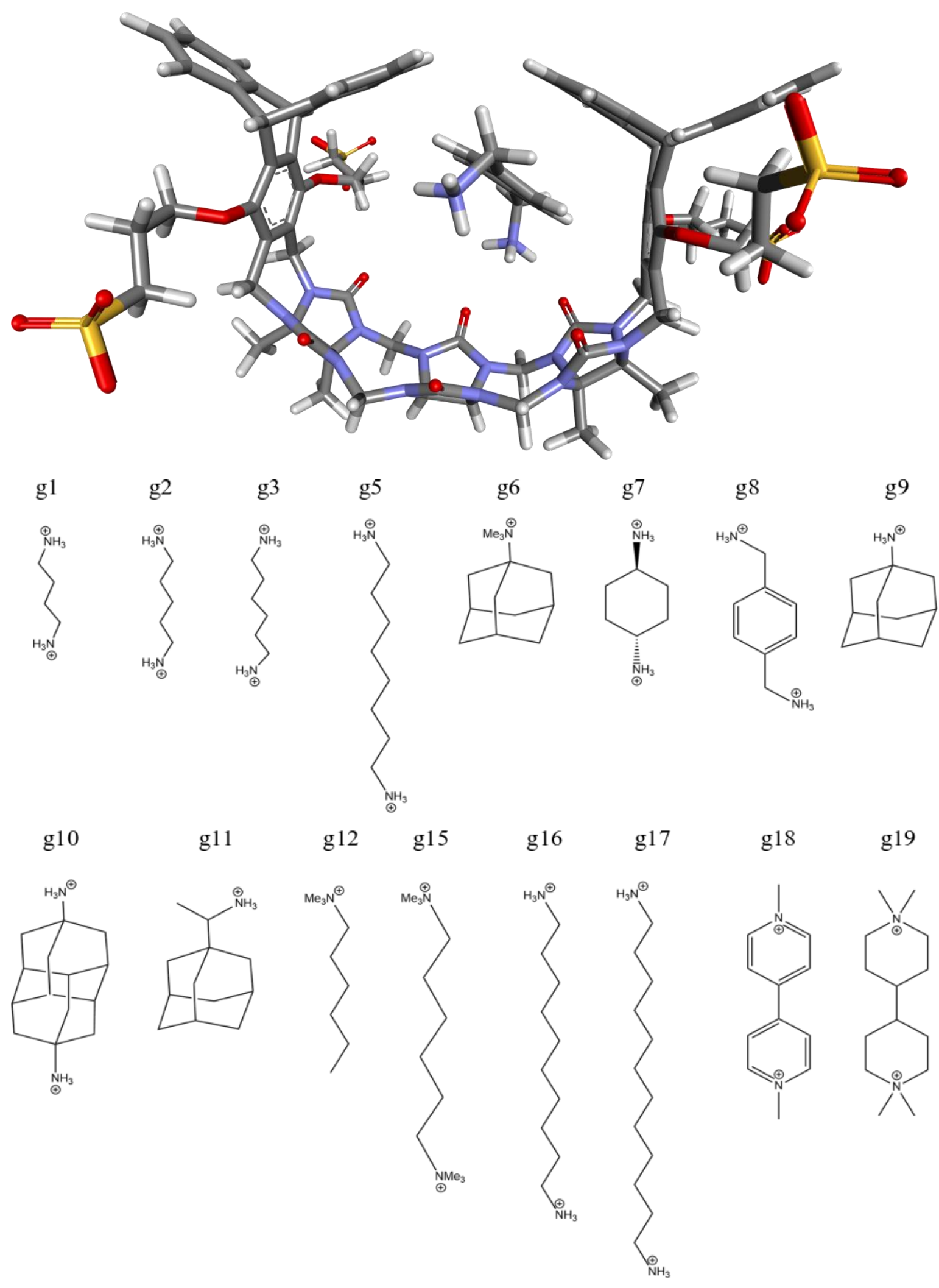

Fig. 1. An illustration of the host-guest binding and the 16 guests of the host TrimerTrip. The host-g8 complex is used as the reference structure in the alchemical free energy calculation and thus is used to generate the picture. The guest $\mathrm{g} 8$ is used as the reference ligand in the relative free energy calculation. 

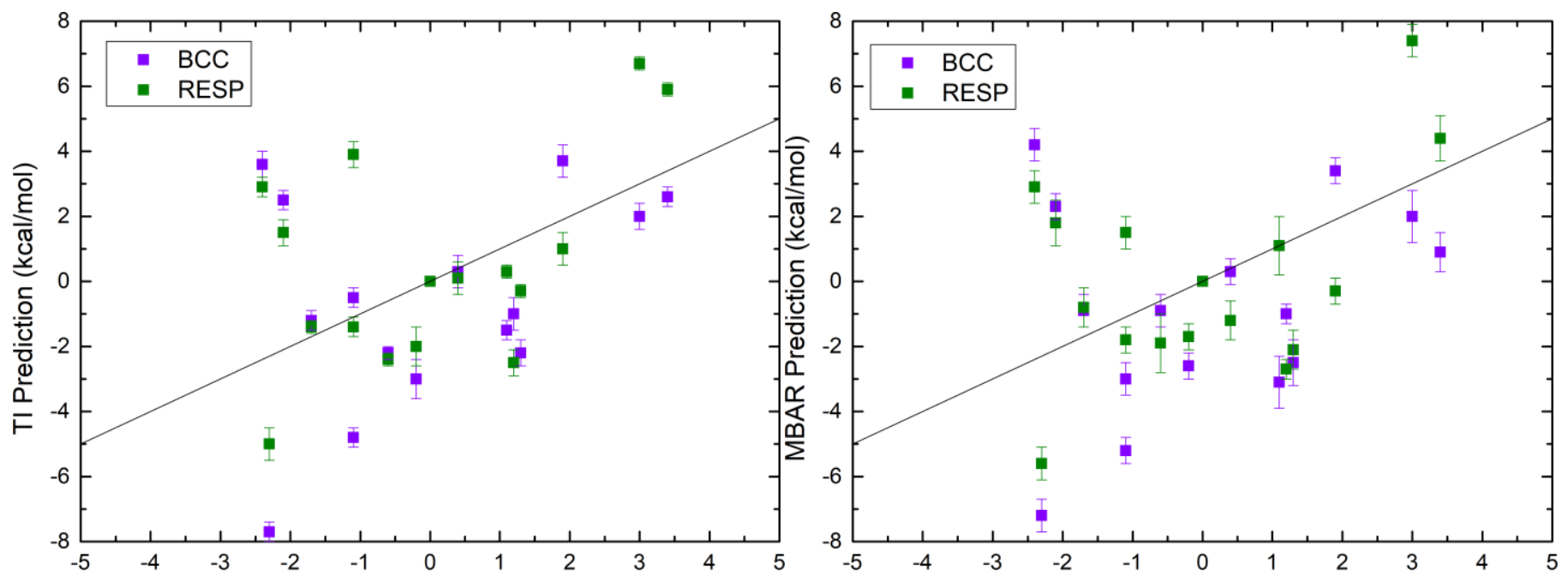

a)

Experiment (kcal/mol)

b)

Experiment (kcal/mol)
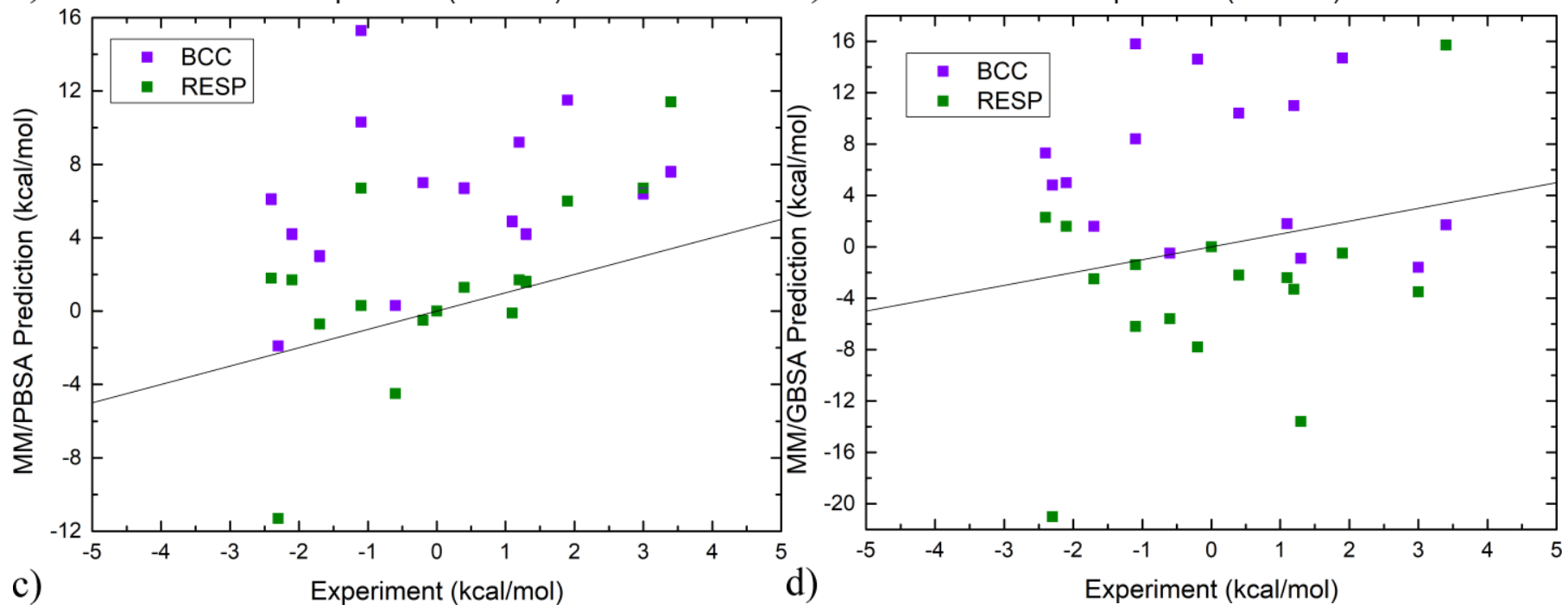

Fig. 2. Comparison between predictions with the AM1-BCC charge scheme and the RESP charges. a) TI, b) MBAR, c) MM/PBSA, d) MM/GBSA. 


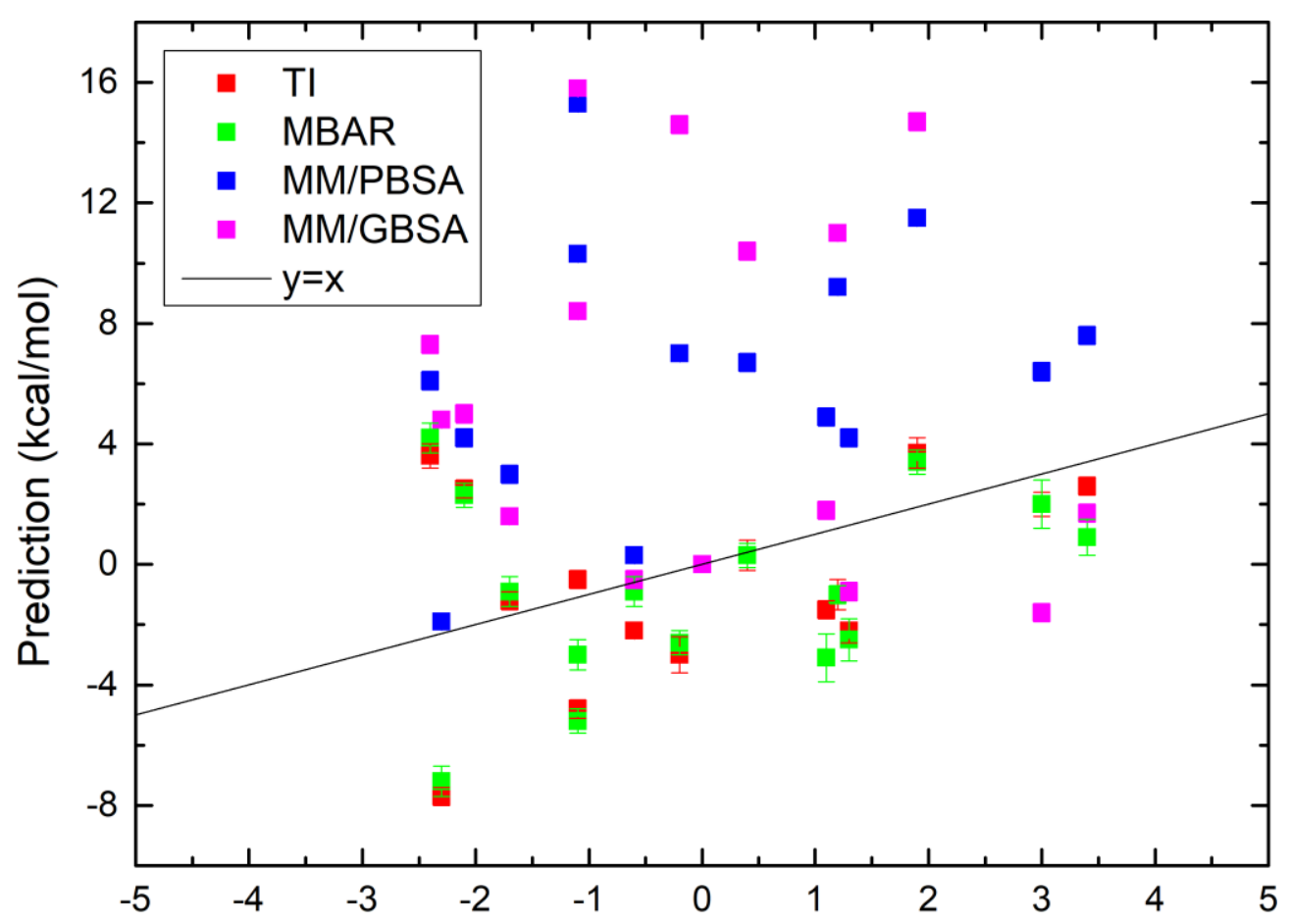

a) $\mathrm{AM1}-\mathrm{BCC} \quad$ Experiment $(\mathrm{kcal} / \mathrm{mol})$

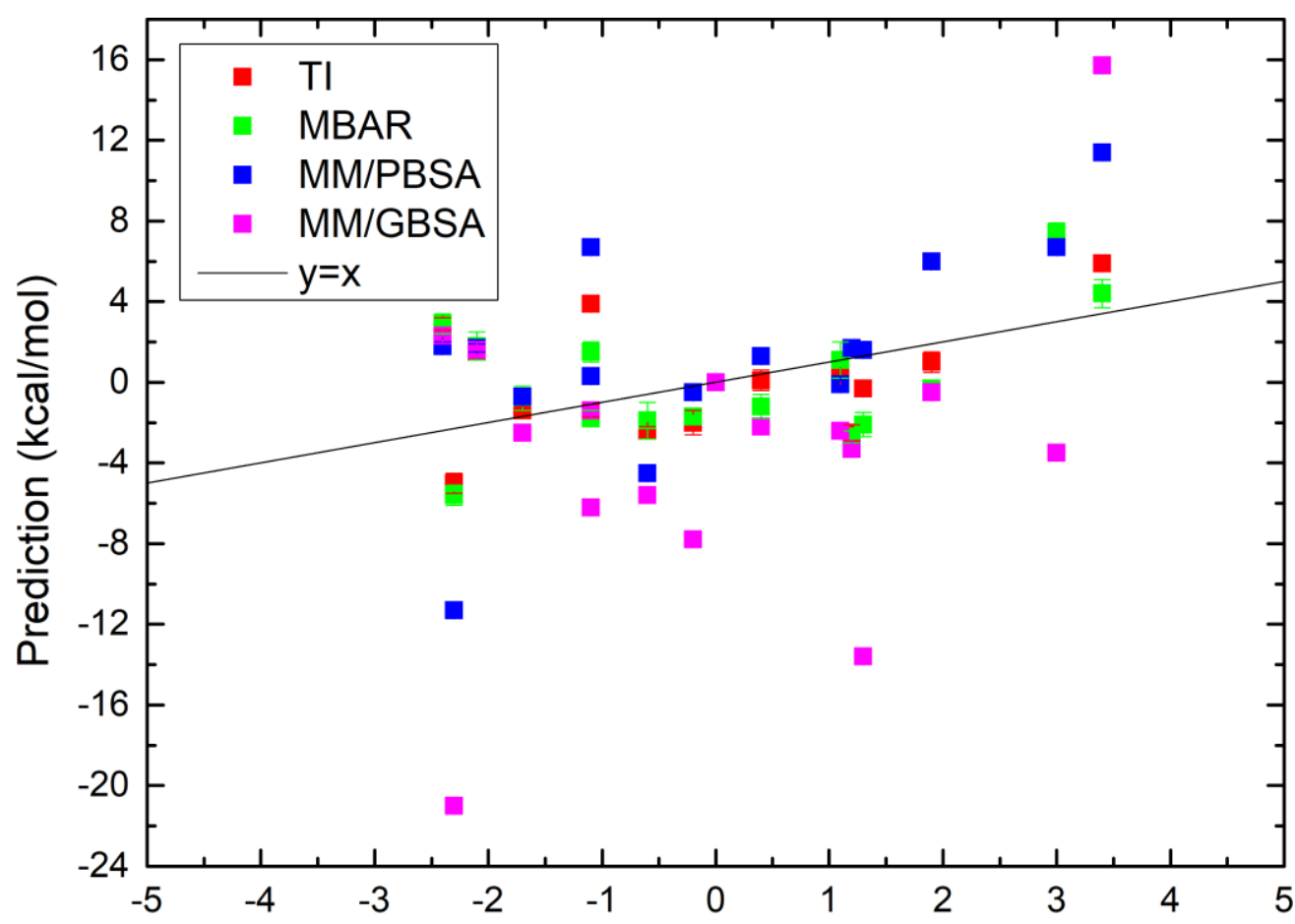

b) RESP

Experiment $(\mathrm{kcal} / \mathrm{mol})$

Fig. 3. Correlation between the predicted binding free energies and the experimental references for TrimerTrip-guest systems constructed with a) AM1-BCC charges and b) RESP charges. The exact values of the binding affinities are presented in Table 1 and 2. 


\section{Supporting Information: SAMPL7 TrimerTrip host-guest binding affinities from extensive alchemical and end-point free energy calculations}

Zhe Huai ${ }^{1}$, Huaiyu Yang ${ }^{2}$, Xiao $\mathrm{Li}^{3}$, and Zhaoxi Sun ${ }^{1,4^{*}}$

${ }^{I}$ State Key Laboratory of Precision Spectroscopy, School of Physics and Electronic Science, East China Normal University, Shanghai 200062, China

${ }^{2}$ College of Engineering, Hebei Normal University, Shijiazhuang 050024, China

${ }^{3}$ Physics, engineering, earth, environment, mechanics (PhITEM), University Grenoble Alpes, 38000 Grenoble, France

${ }^{4}$ Beijing National Laboratory for Molecular Sciences, Institute of Theoretical and Computational Chemistry, College of Chemistry and Molecular Engineering, Peking University, Beijing 100871, China

*To whom correspondence should be addressed: z.sun@pku.edu.cn 
Fig. S1. Time evolution of the relative binding affinities obtained with alchemical free energy calculations different charge schemes: a) AM1-BCC and b) RESP.

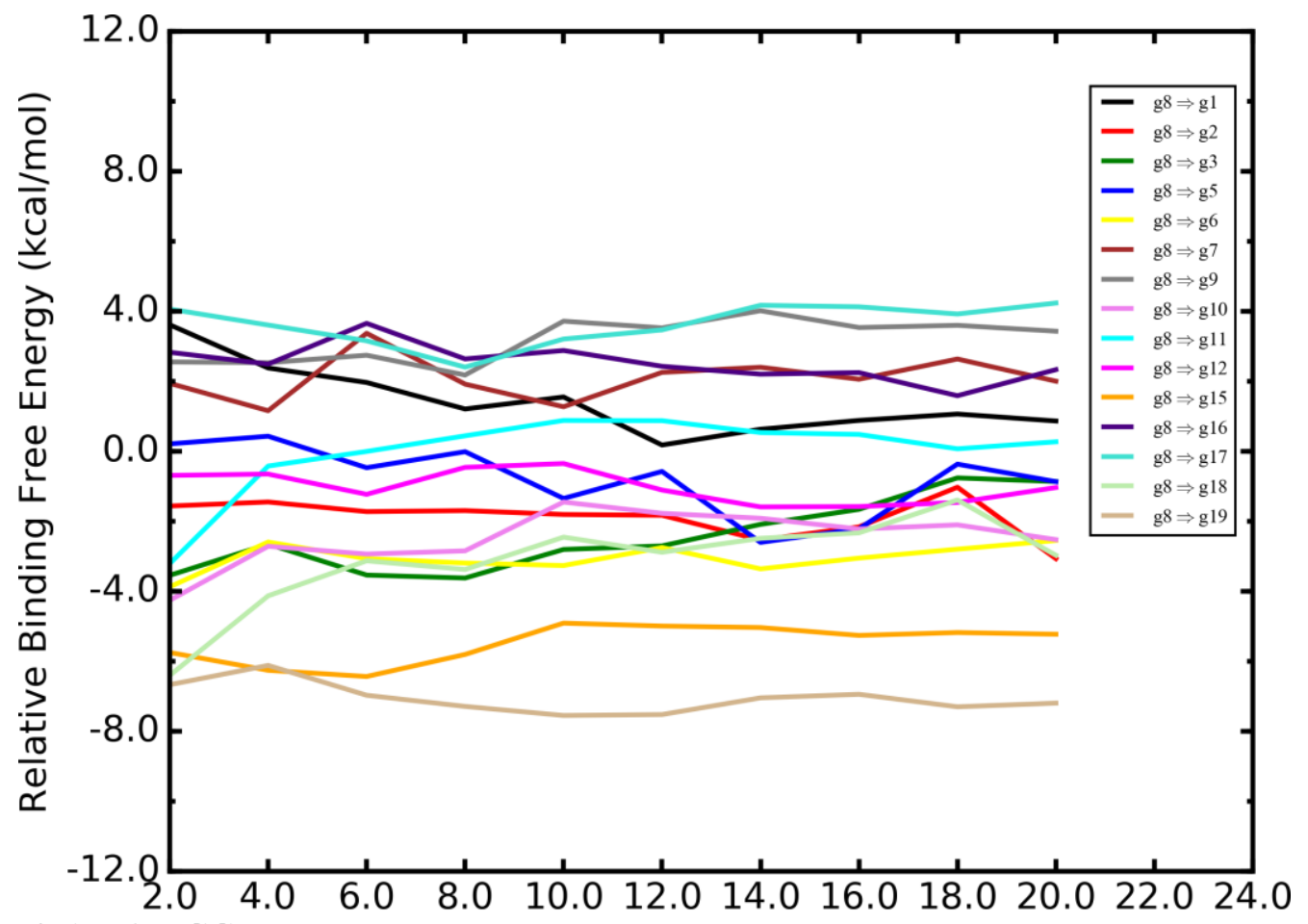

a) AM1-BCC Sampling Time in each Intermediate (ns)

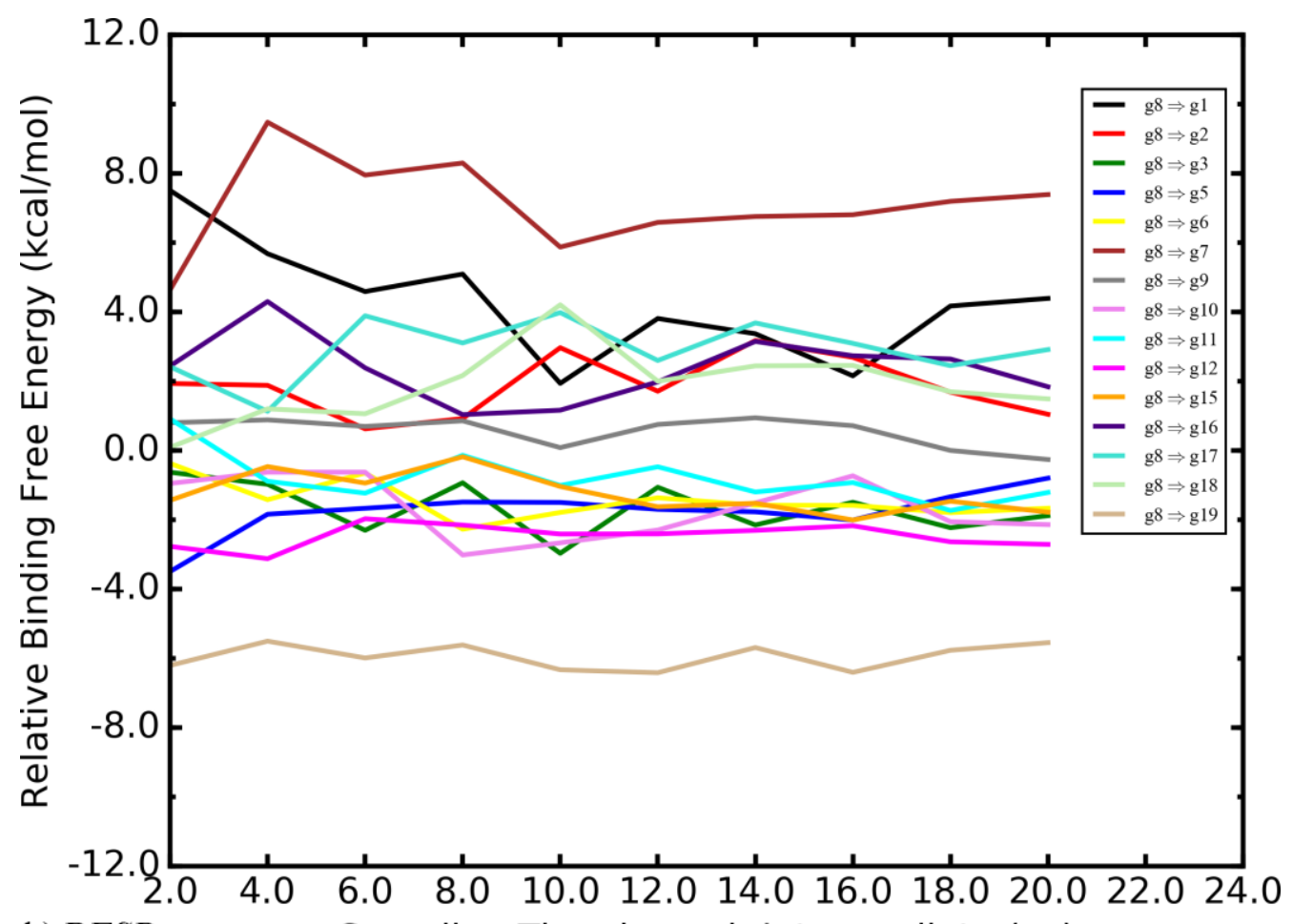

b) RESP Sampling Time in each Intermediate (ns) 
Table S1. Detailed free energy components from MM/PBSA and MM/GBSA with AM1-BCC charges. $\Delta H_{\text {gas }}$ is the gas-phase enthalpy change upon binding or the protein-ligand interaction energy. $\Delta G_{\text {sol }}$ is the solvation free energy and the subscripts of PB and GB denote the implicit solvent model used to calculate the solvation free energy. $\Delta \Delta G_{\mathrm{MM} / \mathrm{PBSA}}$ and $\Delta \Delta G_{\mathrm{MM} / \mathrm{GBSA}}$ are the relative binding affinity with the guest $\mathrm{g} 8$ as the reference.

\begin{tabular}{|c|c|c|c|c|c|c|c|c|c|c|c|c|c|c|}
\hline Guest & $\Delta H_{g a s}$ & SD & $\Delta G_{s o l, P B S A}$ & SD & $\Delta G_{s o l, G B S A}$ & SD & $\Delta G_{\mathrm{MM} / \mathrm{PBSA}}$ & SD & $\Delta G_{\mathrm{MM} / \mathrm{GBSA}}$ & SD & $\Delta \Delta G_{\mathrm{MM} / \mathrm{PBSA}}$ & $\mathrm{SD}$ & $\Delta \Delta G_{\mathrm{MM} / \mathrm{GBSA}}$ & SD \\
\hline $\mathrm{g} 1$ & -469.7 & 1.1 & 451.3 & 1.0 & 426.2 & 1.0 & -18.4 & 0.2 & -43.5 & 0.2 & 7.6 & 0.3 & 1.7 & 0.3 \\
\hline g2 & -468.2 & 1.2 & 447.1 & 1.0 & 424.8 & 1.0 & -21.1 & 0.2 & -43.4 & 0.2 & 4.9 & 0.3 & 1.8 & 0.3 \\
\hline g3 & -461.8 & 0.9 & 436.0 & 0.8 & 416.1 & 0.8 & -25.7 & 0.1 & -45.7 & 0.2 & 0.3 & 0.2 & -0.5 & 0.3 \\
\hline g5 & -451.3 & 0.9 & 428.2 & 0.8 & 407.7 & 0.8 & -23.0 & 0.2 & -43.6 & 0.2 & 3.0 & 0.3 & 1.6 & 0.3 \\
\hline g6 & -227.6 & 0.3 & 208.6 & 0.3 & 197.1 & 0.3 & -19.0 & 0.1 & -30.6 & 0.1 & 7.0 & 0.2 & 14.6 & 0.2 \\
\hline g7 & -474.7 & 1.3 & 455.1 & 1.1 & 428.0 & 1.1 & -19.6 & 0.3 & -46.8 & 0.2 & 6.4 & 0.3 & -1.6 & 0.3 \\
\hline g8 & -468.4 & 1.0 & 442.4 & 0.9 & 423.2 & 0.8 & -26.0 & 0.2 & -45.2 & 0.2 & 0.0 & 0.0 & 0.0 & 0.0 \\
\hline g9 & -225.6 & 0.6 & 211.2 & 0.5 & 195.1 & 0.5 & -14.5 & 0.1 & -30.5 & 0.2 & 11.5 & 0.2 & 14.7 & 0.3 \\
\hline $\mathrm{g} 10$ & -450.6 & 1.3 & 428.8 & 1.1 & 404.5 & 1.1 & -21.8 & 0.2 & -46.1 & 0.2 & 4.2 & 0.3 & -0.9 & 0.3 \\
\hline g11 & -241.9 & 0.7 & 222.6 & 0.6 & 207.1 & 0.6 & -19.3 & 0.2 & -34.8 & 0.2 & 6.7 & 0.3 & 10.4 & 0.3 \\
\hline $\mathrm{g} 12$ & -231.1 & 0.4 & 214.3 & 0.3 & 196.9 & 0.4 & -16.8 & 0.1 & -34.2 & 0.1 & 9.2 & 0.2 & 11.0 & 0.2 \\
\hline g15 & -422.8 & 0.8 & 407.1 & 0.7 & 393.4 & 0.8 & -15.7 & 0.1 & -29.4 & 0.1 & 10.3 & 0.2 & 15.8 & 0.2 \\
\hline $\mathrm{g} 16$ & -423.8 & 0.8 & 402.0 & 0.7 & 383.6 & 0.7 & -21.8 & 0.2 & -40.2 & 0.2 & 4.2 & 0.3 & 5.0 & 0.3 \\
\hline g17 & -396.4 & 0.9 & 376.5 & 0.8 & 358.5 & 0.8 & -19.9 & 0.2 & -37.9 & 0.2 & 6.1 & 0.3 & 7.3 & 0.3 \\
\hline g18 & -441.7 & 0.9 & 431.0 & 0.8 & 404.9 & 0.8 & -10.7 & 0.1 & -36.8 & 0.1 & 15.3 & 0.2 & 8.4 & 0.2 \\
\hline g19 & -466.4 & 0.6 & 438.5 & 0.5 & 426.0 & 0.6 & -27.9 & 0.1 & -40.4 & 0.1 & -1.9 & 0.2 & 4.8 & 0.2 \\
\hline
\end{tabular}


Table S2. Detailed free energy components from MM/PBSA and MM/GBSA with RESP charges. $\Delta H_{g a s}$ is the gas-phase enthalpy change upon binding or the protein-ligand interaction energy. $\Delta G_{s o l}$ is the solvation free energy and the subscripts of PB and GB denote the implicit solvent model used to calculate the solvation free energy. $\Delta \Delta G_{\mathrm{MM} / \mathrm{PBSA}}$ and $\Delta \Delta G_{\mathrm{MM} / \mathrm{GBSA}}$ are the relative binding affinity with the guest $\mathrm{g} 8$ as the reference.

\begin{tabular}{ccccccccccccccc}
\hline Guest & $\Delta H_{\text {gas }}$ & $\mathrm{SD}$ & $\Delta G_{\text {sol }, P B S A}$ & $\mathrm{SD}$ & $\Delta G_{\text {sol }, G B S A}$ & $\mathrm{SD}$ & $\Delta G_{\mathrm{MMM} / \mathrm{PBSA} A}$ & $\mathrm{SD}$ & $\Delta G_{\mathrm{MM} / \mathrm{GBSA}}$ & $\mathrm{SD}$ & $\Delta \Delta G_{\mathrm{MM} / \mathrm{PBSA}}$ & $\mathrm{SD}$ & $\Delta \Delta G_{\mathrm{MM} / \mathrm{GBSA}}$ & $\mathrm{SD}$ \\
\hline $\mathrm{g} 1$ & -416.1 & 1.1 & 408.1 & 1.0 & 393.8 & 1.0 & -8.0 & 0.1 & -22.3 & 0.1 & 11.4 & 0.2 & 15.7 & 0.2 \\
$\mathrm{~g} 2$ & -455.3 & 0.8 & 433.9 & 0.8 & 412.9 & 0.7 & -19.5 & 0.2 & -40.5 & 0.2 & -0.1 & 0.2 & -2.4 & 0.2 \\
$\mathrm{~g} 3$ & -462.5 & 1.0 & 438.7 & 0.9 & 418.9 & 0.9 & -23.8 & 0.1 & -43.7 & 0.1 & -4.5 & 0.2 & -5.6 & 0.2 \\
g5 & -445.1 & 0.8 & 425.1 & 0.7 & 404.5 & 0.7 & -20.0 & 0.1 & -40.6 & 0.1 & -0.7 & 0.2 & -2.5 & 0.2 \\
g6 & -229.5 & 0.3 & 209.7 & 0.3 & 183.7 & 0.3 & -19.9 & 0.1 & -45.8 & 0.1 & -0.5 & 0.2 & -7.8 & 0.2 \\
g7 & -442.8 & 0.6 & 430.2 & 0.6 & 401.3 & 0.5 & -12.7 & 0.1 & -41.6 & 0.1 & 6.7 & 0.2 & -3.5 & 0.2 \\
g8 & -458.8 & 1.0 & 439.5 & 0.9 & 420.8 & 0.8 & -19.3 & 0.2 & -38.1 & 0.2 & 0.0 & 0.0 & 0.0 & 0.0 \\
g9 & -222.4 & 0.4 & 209.0 & 0.4 & 183.8 & 0.3 & -13.4 & 0.1 & -38.6 & 0.2 & 6.0 & 0.2 & -0.5 & 0.2 \\
g10 & -451.8 & 1.0 & 434.1 & 0.9 & 400.2 & 0.8 & -17.7 & 0.2 & -51.6 & 0.2 & 1.6 & 0.3 & -13.6 & 0.3 \\
g11 & -235.3 & 0.6 & 217.3 & 0.5 & 195.1 & 0.5 & -18.0 & 0.2 & -40.2 & 0.2 & 1.3 & 0.2 & -2.2 & 0.2 \\
g12 & -235.3 & 0.3 & 217.7 & 0.3 & 194.0 & 0.3 & -17.6 & 0.1 & -41.4 & 0.2 & 1.7 & 0.2 & -3.3 & 0.2 \\
g15 & -435.7 & 0.8 & 426.6 & 0.7 & 391.4 & 0.7 & -19.1 & 0.1 & -44.3 & 0.2 & 0.3 & 0.2 & -6.2 & 0.2 \\
g16 & -417.0 & 0.9 & 399.3 & 0.8 & 380.5 & 0.8 & -17.6 & 0.1 & -36.5 & 0.1 & 1.7 & 0.2 & 1.6 & 0.2 \\
g17 & -390.8 & 0.9 & 373.2 & 0.8 & 355.0 & 0.8 & -17.6 & 0.1 & -35.8 & 0.1 & 1.8 & 0.2 & 2.3 & 0.2 \\
g18 & -448.7 & 0.7 & 436.1 & 0.6 & 409.2 & 0.6 & -12.6 & 0.1 & -39.5 & 0.1 & 6.7 & 0.2 & -1.4 & 0.2 \\
g19 & -474.8 & 0.6 & 444.2 & 0.5 & 415.7 & 0.5 & -30.6 & 0.1 & -59.0 & 0.1 & -11.3 & 0.2 & -21.0 & 0.2 \\
\hline
\end{tabular}

